- technologischen Determinismus, so HansWerner Ludwig in seinen Ausführungen, sondern bettet die Medien in differenzierte, kulturell weit aufgespannte Kontexte ein. Ludwig wirft höchst interessante Schlaglichter auf gerne vergessene Fakten und Entwicklungslinien im Zusammenhang mit dem Medium Fernsehen und macht deutlich, dass bereits in Bezug auf dieses Medium viel stärker mit Präzisierungen und Differenzierungen gearbeitet werden müsse. Die vom Autor angeführte These von der zunehmenden Emotionalisierung und Reizsteigerung hätte sich wunderbar mit den zentralen Gedanken McLuhans zum Unbewussten und zur Synästhesie der Medien verbinden lassen. Eine Rückbindung an die Ausführungen McLuhans wird hier aber ebenso versäumt wie die Chance für eine dezidiert vorgebrachte Kritik.

Betrachtet man die Gesamtheit der Aufsätze des vorliegenden Bandes, ahnt man was sich im Feld der Medienrhetorik - ein im übrigen noch nicht einmal eingeführter Begriff, der davon ausgeht, dass Medien „vermutlich ein rhetorisches Potential haben, also dass Medien in ihrer Eigenschaft als Medien rhetorische Effekte entfalten “ (S. 7) - an offenen Fragen und zu beforschenden Gebieten auftut. Ebenso deutlich wird, dass es sich nach wie vor lohnt, McLuhan einer (kritischen) Relektüre zu unterziehen bzw. seine Gedanken und Ausführungen zu aktualisieren.

\section{Daniela Kloock}

\section{Hans-Jürgen Lüsebrink}

\section{Interkulturelle Kommunikation}

Interaktion, Fremdwahrnehmung, Kulturtransfer

Stuttgart: Metzler, 2005. - 211 S.

ISBN 3-476-01989-6

Interkulturelle Kommunikation, die Kommunikation zwischen Menschen unterschiedlicher Kulturen, ist in Zeiten von Kriegen, Terrorismus und Konflikten, die immer wieder mit unvereinbaren kulturellen Gegensätzen begründet werden, nötiger denn je, wird aber in der deutschen kommunikationswissenschaftlichen Forschung wenig beachtet. Umso erfreulicher ist es, den Band „Interkulturelle Kommunikation“ von Hans-Jürgen Lüsebrink zu sehen, der das Themainterdisziplinär aus verschiedenen Blickwin- keln betrachtet. Das Buch ist als Einführung in Fragestellungen, Methoden und Gegenstandsbereiche der interkulturellen Kommunikation gedacht. Ziel ist es, neben Aktualitätsbezügen vor allem die historische Dimension kultureller Prozesse zu beleuchten. Ausgangspunkt sind hier Erfahrungen und Anforderungen der Studiengänge „Französische Kulturwissenschaft und Interkulturelle Kommunikation" sowie „Grenzüberschreitende Deutsch-Französische Studien “ der Universität des Saarlandes, an der der Autor forscht und lehrt.

Diesem Standpunkt ist es zu verdanken, dass nicht nur die bekannte deutsch- und englischsprachige Literatur berücksichtigt wird, sondern Publikationen aus dem gesamten frankophonen Raum Beachtung gefunden haben. Gerade die frankoafrikanische und frankokanadische Literatur eröffnet dem Leser immer wieder interessante Einblicke. Fremdwahrnehmungsmuster, zum Beispiel in dem französischen Diskurs über Afrika oder dem kanadischen über die Indianerkulturen Nordamerikas, verglichen mit dem deutschen Diskurs über Amerika zeigen im Rahmen der Diskursanalyse, dass diese Muster nicht isoliert gesehen werden können, sondern einer vielschichtigen Auswertung von Bezugsnetzen bedürfen.

Die Anlage ist breit: Nach einer allgemeinen Einordnung der interkulturellen Kommunikation werden Konzepte wie Multikulturalität, Transkulturalität und Hybridität vorgestellt und Problemfelder der interkulturellen Kommunikation aufgezeigt. Konfrontationen zwischen Menschen verschiedener Kulturen und kulturelle Konflikte stehen dabei im Mittelpunkt: Verstehen von Kultur durch den Vergleich von Texten wird hier als produktiver Lösungsweg aufgezeigt.

Im anschließenden Kapitel „Interaktion“ werden verschiedene Formen der empirischen Analyse vorgestellt: interkulturelle neben kulturkontrastiven, psychologische neben linguistischen Ansätzen. Nachdem interkulturelle „Problem-Kommunikation“ auf Makro- und Mikroebene analysiert wird, erfolgt eine Überleitung zu konkreten Anwendungsbereichen: zum einen zum Bereich Interkulturelles Lernen, einem international sehr intensiv diskutierten Problemfeld des Bildungsbereichs, zum anderen zum Bereich der interkulturellen Trainingsformen und -methoden, die im Zuge der Globalisierung von Unternehmen und dem weltweiten mobilen Einsatz ihrer Mitarbei- 
ter zunehmend an Bedeutung gewinnen. Hier werden Aspekte, die bei der traditionellen Beschäftigung der Kommunikationswissenschaft mit Massenmedien eher unbeachtet bleiben, in den Mittelpunkt gerückt. Dies ist ein Beispiel für die Verzahnung von Theorie und Praxis auf der Ebene der interkulturellen Kommunikation. Für die Absolventen von Studiengängen mit interkulturellem Schwerpunkt sind diese konkreten Anwendungsbeispiele interessant, da sich hier Berufsperspektiven finden lassen.

Im dritten Teil, den „Fremdwahrnehmungen“, gibt der Autor einen Überblick über Ansätze, mit dem Eigenen und dem Fremden umzugehen, wobei neben den kulturanthropologischen Dimensionen und interkulturellen Interaktionssituationen auch die aktuelle Wahrnehmung des Fremden in den Medien berücksichtigt wird.

Medien werden ebenfalls im letzten Teil thematisiert, in dem der Kulturtransfer behandelt wird. Auch in diesem Teil gelingt es dem Autor, Kulturtransferprozesse anhand von vielen Praxisbeispielen plastisch zu verdeutlichen, sowohl in Bezug auf historische als auch auf aktuelle Ansätze. Mit Beispielen aus dem Gebiet des interkulturellen Marketings, die unter anderem unterschiedliche Kommunikations- und Werbestile illustrieren, wird erneut der Bogen zwischen Theorie und Praxis der interkulturellen Kommunikation geschlagen. Medialer Kulturtransfer oder auch interkulturelle Rezeption verschiedener Medienangebote werden anhand von Fallbeispielen aufgezeigt, beispielsweise amerikanische Adaptionen französischer Filme, wie Jim McBrides Neuverfilmung „Breathless“, der Jean-Luc Godards Film „A bout de souffle" zur Vorlage nimmt.

Im letzten Teil zieht der Autor Bilanz über die Perspektiven der interkulturellen Kommunikation. Die Globalisierung stellt auch hier neue Herausforderungen: Immigration und Multikulturalität zeigen Felder auf, in denen Kulturen aufeinander treffen, vermittelnde Kommunikation aber kaum stattfindet und oft sogar rückläufig ist. Dies gilt gerade für Deutschland, das im internationalen Vergleich nur über eine rudimentäre koloniale Geschichte verfügt. Als Philologe fordert der Autor vor allem die Einbeziehung von Sprache bei der Untersuchung von Kulturtransferprozessen und Fremdwahrnehmungsmustern in die interkulturelle Forschung.

Lüsebrink hat ein Werk vorgelegt, das sehr positiv auffällt durch die Interdisziplinarität bei der Herangehensweise an das Forschungsfeld der interkulturellen Kommunikation. Breit gefächert verbindet er verschiedene Wissenschaftsdisziplinen miteinander, wie Anthropologie, Betriebswirtschaftslehre und Kulturwissenschaft. Erstaunlich ist, dass die Disziplin der Kommunikationswissenschaft kaum herangezogen wird, neuere theoretischen Ansätze wie die transkulturelle Kommunikation wurden nicht rezipiert. „Interkulturelle Kommunikation" ist ein Buch, das den Blick öffnet für neue Sichtweisen auf aktuelle interkulturelle Problemfelder, die oft eine historische Dimension haben, welche aber - wie der Autor zu Recht feststellt - meist vergessen wird.

Sonja Kretzschmar

\section{Harald Gapski (Hrsg.)}

\section{Medienkompetenzen messen?}

Verfahren und Reflexionen zur Erfassung von Schlüsselkompetenzen

Düsseldorf/München: kopaed 2006. - 136 S.

(Schriftenreihe Medienkompetenz des Landes Nordrhein-Westfalen; Bd. 3)

ISBN 3-938028-53-X

Lassen sich Medienkompetenzen messen oder zunächst einmal hinreichend eindeutig definieren und, wenn ja, wie? So lautet das etwas amputiert annoncierte Thema dieses Readers, der auf einen „Expertenworkshop“ des ecmc, des Europäischen Zentrums für Medienkompetenz in Marl, im November 2005 rekurriert. Nein, hieße eigentlich die aufrichtige Antwort, oder allenfalls: es kommt darauf an, wie die diversen Kontexte, die Ziele, Normen, Inhalte und Zielgruppen ausfallen, aber auch darauf, ob Medienkompetenz als individuelle Fähigkeit, als strukturelle einer Institution oder eines Systems - wie immer sie dann gekennzeichnet und identifiziert wird bzw. werden soll-, ob sie als intentionaler Zielhorizont, als weitgehend informeller Prozess oder als testierbare Eigenschaft gesehen wird. Dazu geben die hier versammelten sechs Beiträge Begründungen, Beispiele und methodische Vorschläge, wobei sie sich eher mit allgemeinen Fragen beschäftigen, mithin selten in das Kernfeld der Medienkompetenz vorstoßen, eher methodische Optionen vorschlagen als dass sie konkrete Messoperationen vorstellen. So bleibt die im Vorwort geäußerte Hoffnung 